\title{
Elevated Frequency Loading of Facet Joints
}

\author{
Antonio Valdevit ${ }^{1,2}$, Emily Noonan', Hannah Sidoti1 ${ }^{1}$, Rebecca Chung1, Arthur Ritter ${ }^{1}$, \\ Thomas Errico ${ }^{2}$ \\ ${ }^{1}$ Dept. of Chemistry, Chemical Biology, Biomedical Engineering, \\ Stevens Institute of Technology, Hoboken, NJ, 07030 \\ Antonio.Valdevit@stevens.edu; enoonan@stevens.edu;hsidoti@stevens.edu;aritter@stevens.edu \\ ${ }^{2}$ Spine Division, Department of Orthopedics, NYU Langone Medical Center, \\ New York, NY, 10005 \\ spinecore@hotmail.com
}

\begin{abstract}
With disc degeneration and height loss, facet joints may become susceptible to damage when exposed to elevated frequency loading. The investigators hypothesized that elevated frequencies alter normal mechanical response of facet joints which may be a mechanical predisposition for low back pain. Facet joints from six bovine L4-L5 vertebra were subjected to 520 loading cycles from $-15 \mathrm{~N}$ to $-60 \mathrm{~N}$ at $1 \mathrm{~Hz}, 5 \mathrm{~Hz}$, $20 \mathrm{~Hz}, 40 \mathrm{~Hz}$ and $80 \mathrm{~Hz}$. Net deformation, strain, cumulative strain, and elastic stiffness were computed for each cycle, averaged across frequencies and subjected to non-linear exponential regression. Regression parameters were examined with a Tukey post-hoc test. Asymptotic limits of deformation were statistically significant $(P<0.005)$ while elevated frequencies displayed significant decreases with respect to deformation change per cycle as compared to $1 \mathrm{~Hz} .(\mathrm{P}<0.005)$ Initial strain data indicated all frequency comparisons were statistically significant $(P<0.01$. The strain change per cycle indicated $1 \mathrm{~Hz}$ loading was statistically equivalent to both $40 \mathrm{~Hz}$ and $80 \mathrm{~Hz}$ loading $(P>0.05)$. The $5 \mathrm{~Hz}$ frequency was statistically elevated compared to other frequencies $(P<0.01)$. Initial stiffness indicated all frequency comparisons were statistically different $(P<0.05)$. This study represents mechanical evidence for the predisposition of individuals exposed to high frequency loading toward increased incidence of load back pain.
\end{abstract}

Keywords: Loading Frequency, Compression. Facet Joint Damage.

(C) Copyright 2015 Authors - This is an Open Access article published under the Creative Commons Attribution License terms (http://creativecommons.org/licenses/by/3.0). Unrestricted use, distribution, and reproduction in any medium are permitted, provided the original work is properly cited.

\section{Introduction and Related Work}

Societal lifestyle and occupational trends combined with natural disc degeneration due to aging results in low back pain affecting over $80 \%$ of Americans at some point in their lives [1]. The facet joints have been recognized as a potential source of back pain since 1911 [2]. While this incidence rate may be relatively high, perhaps a more pertinent observation involves those individuals who are exposed to elevated frequency loading due to occupation. These include heavy equipment operators, commercial truck drivers and helicopter pilots. A meta-risk analysis for development of low back pain in this population indicated a propensity over twice that of non-operators [3]. In animals, complications due to vibrational loading include compromised nutrition, increased disc pressure, neuropeptide release, and increased creep [4]. Feline models subjected to 60 minutes of cyclic flexionextension at $40 \mathrm{~N}$ at $0.25 \mathrm{~Hz}$ and $0.5 \mathrm{~Hz}$ respectively, resulted in significant increase in pro-inflammatory cytokines (IL-1 $\beta$, IL-6, IL-8, TNF- $\alpha$, TGF- $\beta 1$ ) at the higher loading frequency, indicating pronounced changes consistent with an acute inflammation in the supraspinous ligaments of L3-L4 and L5-L6 [5]. In humans, vibrational exposure has been related to low back pain in addition to neck and shoulder pain. [6].

Under certain conditions the effects of high frequency vibration has been shown to provide benefit. Rats bilaterally implanted with hydroxyapatite (HA)coated titanium in tibiae were treated with lowmagnitude high-frequency loading via whole body vibration (VIB) or alendronate (ALN) following ovariectomy. Both the VIB, ALN and sham animals 
significantly increased bone-to-implant contact, periimplant bone fraction and biomechanical push out force and interfacial shear strength when compared with the control ovariectomy group. The authors concluded that low-magnitude high-frequency loading enhanced boneto-implant osseointegration, but the efficacy was weaker than alendronate [7]. Though a number of studies have been conducted to evaluate vibrational transmission to the body, few have focused on isolated spinal structures. One such study by Kaigle et al. investigated the response of porcine intervertebral discs to compressive $(100 \mathrm{~N}$ and $200 \mathrm{~N})$ vibrational loading from $0.05 \mathrm{~Hz}$ to $25 \mathrm{~Hz}$. The results showed that dynamic axial stiffness was frequency dependent and increased with successive exposures at the same load magnitude [8].

Finite element analyses have been used extensively to examine the effects upon spinal structures. Using a non-linear finite element model of the L3-L5 spinal segment, Guo et al. examined the frequency effects of axial cyclic loading on the spine. When exposed to long term whole body vibration, the model indicated that the posterior regions of intervertebral disc are more susceptible to injury as compared to anterior regions. Further, the vibrational amplitudes displayed at the facet joint were in excess of $200 \%$ of both the displacement and stress on vertebrae or discs [9]. Regarding implant performance, Kettler et al. investigated the wear rates of Prodisc-L devices tested under ISO 18192-1 (Wear of total intervertebral spinal disc prostheses -- Part 1: Loading and displacement parameters for wear testing and corresponding environmental conditions for test) and found that testing at $2 \mathrm{~Hz}$ resulted in increased wear as compared to the traditional $1 \mathrm{~Hz}$ testing rate [10].

Several studies have concluded that the resonance frequency of the spine is approximately $5 \mathrm{~Hz}$. $[6,11,12]$. While dynamic evaluations of elevated frequency effects have been conducted few have examined loading frequencies in excess of the resonance frequency [8]. One drawback to whole body vibration is identifying the exact frequency and magnitude of load applied to structures of interest. Through cyclic loading at these elevated frequencies, one may investigate the temporal effects of elevated frequency loading. Furthermore, using this temporal data, extrapolation to prolonged exposure is possible, though it should be noted that under these in-vitro conditions, one must also give some credence to specimen degradation as well as the lack of physiological repair.

In this paper the investigators applied cyclic compression to spinal facet joints over a range of loading frequencies from the physiologically accepted value of $1 \mathrm{~Hz}$ to a high rate of $80 \mathrm{~Hz}$ so as to span frequencies encountered under occupational exposure. We hypothesized that elevated frequency loading changes the mechanical response of the facet joint to loading. In doing so, the results indicated that elevated loading frequencies stiffened the facet joint and indicated a continuous accumulation of strain at frequencies beyond $1 \mathrm{~Hz}$. These findings are mechanical evidence toward a predisposition of those individuals associated with high frequency occupational exposure for increased incidence of low back pain.

\section{Materials and Methods}

Facet joints from six 100kg bovine L4-L5 vertebral bodies (Animal Technologies Inc., Tyler, TX) were embedded in resin and secured to a mechanism that permitted translation and rotation in the X-Y plane to allow perpendicular alignment of the test surface to the loading axis. Each loading site was subjected to 520 loading cycles from $-15 \mathrm{~N}(19 \mathrm{MPa})$ to $-60 \mathrm{~N}(76 \mathrm{MPa})$ using a $1 \mathrm{~mm}$ diameter indentor (Figure 1).

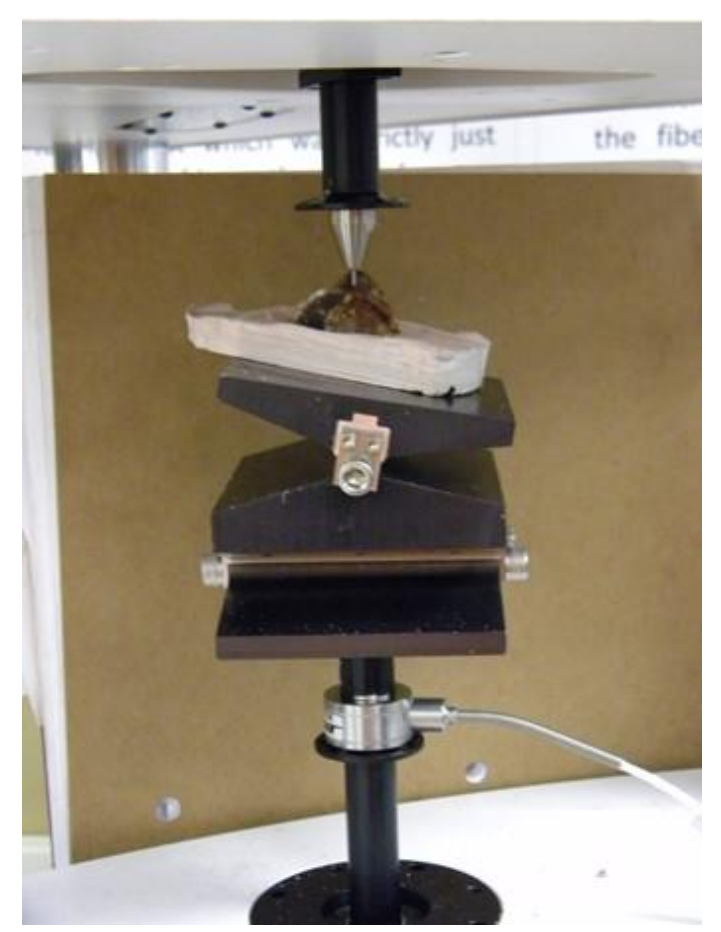

Figure 1. Mechanical loading mechanism that permits X,Y translation and rotation to allow for perpendicular alignment of the loading axis and the contact surface. 
Previous work has shown that continuous loading over extended cycles can lead to specimen degradation. These testing parameters are comparable to those cited by Zioupos et.al, where specimens under $80 \mathrm{MPa}$ were failing at approximately 550 cycles using human cortical bone [13]. Load versus deformation data was recorded at cycle 5 and at subsequent 25-cycle intervals. The central region of the facet was tested at $1 \mathrm{~Hz}$ with surrounding sites at distances of 2 indentor diameters randomly subjected to loading frequencies of $5 \mathrm{~Hz}, 20 \mathrm{~Hz}, 40 \mathrm{~Hz}$ and $80 \mathrm{~Hz}$ [14]. The maximum deformation at each cycle interval was computed and averaged across all specimens for each frequency, initialized to zero at the initial cycle interval and plotted verses cycle number. The stiffness in the elastic region was computed by performing a linear regression for load versus deformation using data from the first complete loading cycle at each cycle count interval.

Stiffness data at each respective cycle count was subsequently averaged across all specimens for each frequency and plotted versus cycle number. For each frequency, the cycle strain was computed and averaged across frequencies for each cycle over all specimens. The cumulative strain versus cycle number data was also computed. All parameters were subjected to nonlinear exponential regression. The resulting regression produced the parameters of Yo (initial net deformation), $\mathrm{K}$ (rate constant of net deformation change), Plateau (asymptotic limit of net deformation) and Span (Yo - Plateau) which were examined using a 1way ANOVA with a Tukey post-hoc test for between frequency comparisons. The $\mathrm{K}$ values for maximum deformation were converted to half-life according to the relationship: half-life $=\ln 2 / \mathrm{K}$. Half-life in this context represents the number of cycles required to attain $50 \%$ of the asymptotic deformation limit or Plateau.

\section{Results}

Figure 2 depicts the net deformation versus cycle number at each frequency. All comparisons for Plateau values were statistically significant between frequencies. ( $\mathrm{P}<0.005$ all comparisons)

The resulting $\mathrm{K}$ value or rate of net deformation per unit cycle at elevated frequencies $(>1 \mathrm{~Hz})$ displayed statistically significant decreases with respect to the $1 \mathrm{~Hz}$ frequency (Figure 3 ).

No statistically significant differences between $\mathrm{K}$ values were detected between $5 \mathrm{~Hz}$ and $40 \mathrm{~Hz}$ or $80 \mathrm{~Hz}$. However the associated $\mathrm{K}$ value with the $20 \mathrm{~Hz}$ frequency was significantly decreased as compared to the $5 \mathrm{~Hz}$ frequency. The $\mathrm{K}$ value associated with the $20 \mathrm{~Hz}$ frequency was significantly different as compared to $80 \mathrm{~Hz}(\mathrm{P}<0.05)$ but not $40 \mathrm{~Hz}$.

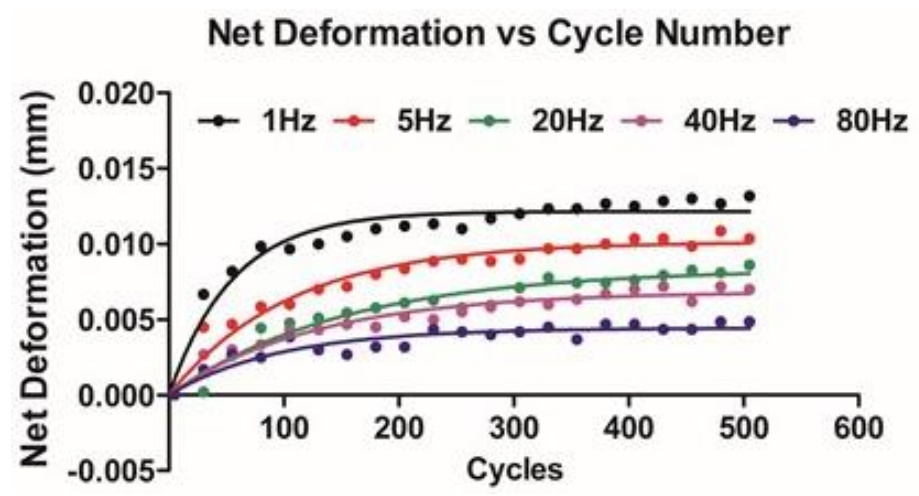

Figure 2. Deformation. Plot of maximum deformation versus cycle number. All frequencies displayed an exponential relationship.

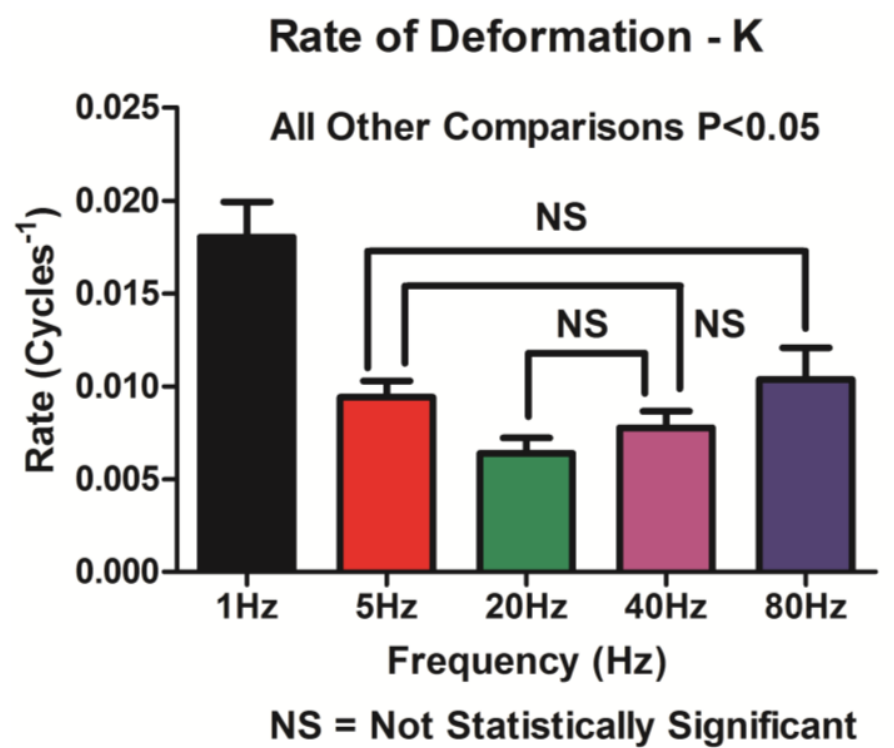

Figure 3. Deformation rate per cycle; K value. All elevated frequencies displayed a statistically reduced $\mathrm{K}$ value as compared to the $1 \mathrm{~Hz}$ frequency.

The asymptotic deformation values or Plateau achieved by the facet surface based on frequency were statistically significant. ( $\mathrm{P}<0.005$ all comparisons) All elevated frequencies $(>1 \mathrm{~Hz})$ displayed statistically significant decreases with respect to $\mathrm{K}(\mathrm{P}<0.05$ over all comparisons). The half-life data for deformation indicates that the $5 \mathrm{~Hz}$ is statistically decreased as compared to all other frequencies tested (Figure 4).

The regression results of stiffness versus cycle number also indicated an exponential relationship. Both the initial stiffness (Yo) and asymptotic limit of the 
stiffness (Plateau) increased as the frequency increased with all comparisons between frequencies statistically significant $(\mathrm{P}<0.05$ for all). With respect to the Half-Life stiffness data, no statistically significant differences were found between the $5 \mathrm{~Hz}$ and $20 \mathrm{~Hz}$, or the $1 \mathrm{~Hz}$ and $80 \mathrm{~Hz}$ frequencies. All other comparisons were statistically significant $(\mathrm{P}<0.05)$.

A decrease in the $\mathrm{K}$ value (that is, an increased number of cycles to obtain the asymptotic stiffness limit) was seen as the frequency increased from $1 \mathrm{~Hz}$ to $40 \mathrm{~Hz}$. At the $80 \mathrm{~Hz}$ loading frequency, the $\mathrm{K}$ value was comparable to that observed at the $1 \mathrm{~Hz}$ loading rate.

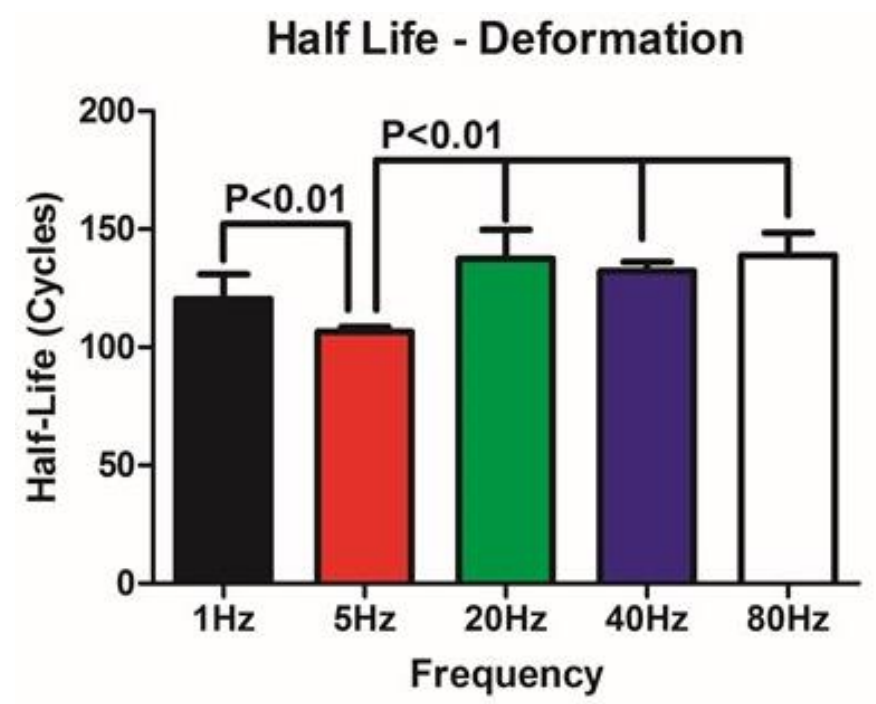

Figure 4. Deformation Half-Life or number of cycles required to achieve a $50 \%$ reduction in deformation. The $5 \mathrm{~Hz}$ or cited resonant frequency displayed a reduced number of cycles as compared to all other frequencies.

The cycle strain indicated that both the initial and asymptotic limit of the strain decreased with increasing frequency $(\mathrm{P}<0.05$ for all comparisons). The $\mathrm{K}$ value for strain (or rate of strain change per cycle) indicated that the $1 \mathrm{~Hz}$ loading frequency was statistically equivalent to both the $40 \mathrm{~Hz}$ and $80 \mathrm{~Hz}$ frequencies ( $\mathrm{P}>0.05$ for both) The $5 \mathrm{~Hz}$ frequency $\mathrm{K}$ value was statistically increased as compared to all other frequencies $(\mathrm{P}<0.01$ for all comparisons)(Figure 5).

For the cumulative strain data, all frequencies above $1 \mathrm{~Hz}$ displayed a linear response between strain accumulation and cycle number whereas at $1 \mathrm{~Hz}$ frequency an exponential relationship was evident $(\mathrm{P}<0.001, \mathrm{~F}$ - test)(Figure 6). A statistical comparison of the linear regression slopes for the elevated frequencies versus the $\mathrm{K}$ value for the $1 \mathrm{~Hz}$ frequency indicated that all elevated rates were statistically different as compared to $1 \mathrm{~Hz}$ loading $(\mathrm{P}<0.001$ for all $)$.

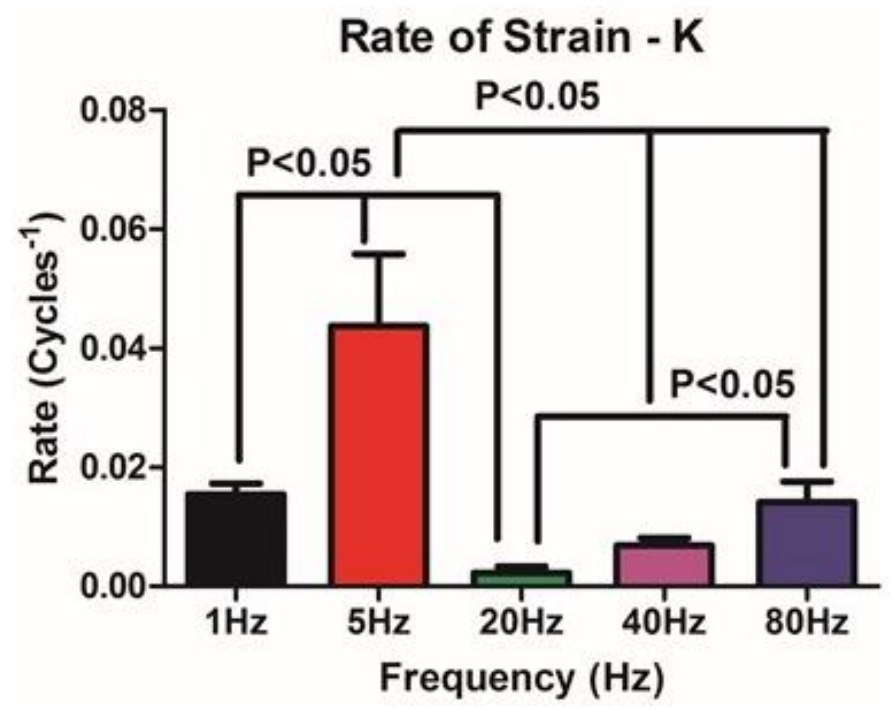

Figure 5. Strain rate per cycle; K value. The $5 \mathrm{~Hz}$ frequency $\mathrm{K}$ values were significantly increased as compared to all other frequencies, indicating a reduction in the number of cycles required to achieve strain stability.

\section{Cycle Cummulative Strain Extrapolated}

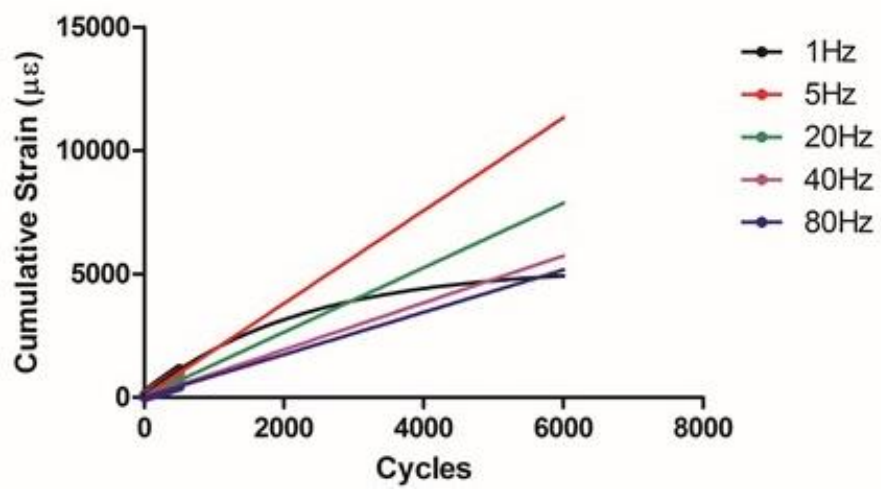

Figure 6. Extrapolated Cumulative Strain. Extrapolation of the fitted cumulative strain equations indicates that at 6000 cycles all elevated frequencies tested accumulated strain at levels above that seen for $1 \mathrm{~Hz}$ frequencies where an asymptote has been achieved.

\section{Discussion}

In a review of the literature concerning elevated frequency loading, Hill et al. determined that while studies displayed deficiencies, vibrations remain an important consideration in evaluation of patients presenting with discogenic back pain and that long term clinically controlled studies will be required for a definitive determination of vibrational effects upon spinal loading [15]. Determination of spinal resonance 
frequency is difficult as the frequency response is affected by multiple factors which include posture, seating, and inclination [6]. Despite variations in experimental methods, the resonance frequency of the spine has been cited between $5 \mathrm{~Hz}$ and $12 \mathrm{~Hz}$ with the greatest transmission occurring at the first resonant frequency of $5 \mathrm{~Hz}[6,15]$.

At these elevated frequencies damping may result in considerable energy transfer. This was validated in this study by statistically increased values in initial and asymptotic stiffness values as loading frequency is elevated (Figure 7).


Figure 7. Stiffness Results. The initial stiffness and asymptotic stiffness values increase as the loading frequency increases. All comparisons were statistically significant.

With increased frequency, increases in initial stiffness and cycles required to achieve stability (i.e. decreasing $\mathrm{K}$ values for stiffness) were seen. This is in keeping with the work by Wilder et al., where stiffening of the spine was reported in response to loading at increasing frequency. Further, it has been found that structures vibrated at the first resonant frequency have greater potential for damage [16]. In this study, a similar finding that $5 \mathrm{~Hz}$ frequency loading was more often found to be associated with more detrimental mechanical parameters as compared to the other elevated frequencies.

In this study, the combination of decreasing $\mathrm{K}$ values (for deformation and stiffness respectively) and elevated initial stiffness may constitute a predisposition in individuals for the onset of facet joint degeneration. This is significant in that viscous damping may result in significant energy transfer. At elevated frequencies muscles become fatigued and require increased time to react to sudden loading thereby making the spine more susceptible to injury [6]. Guo et al. noted that whole body vibration of human spine is more dangerous to facets, which may lead to abnormal remodelling and degeneration of the lumbar spine [9].

Bone, as many viscoelastic tissues, increases in strength with strain rate. While the applied loading is uniform, the reduction in initial strain due to elevation in frequency results in an increased modulus or stiffening. The $40 \mathrm{~Hz}$ and $80 \mathrm{~Hz}$ frequencies may be acting as a low frequency or quasi-static condition in that the fluid flow within the tissue has achieved static equilibrium and hence the viscoelastic effects are comparable to those at low frequency.

The cumulative cycle strain data has clinical implications in that eventual attainment of a plateau level is attained at $1 \mathrm{~Hz}$ while elevated frequencies continue accumulation indefinitely. If one considers the continued exposure of these elevated frequencies by extrapolating to several thousand cycles the evidence suggests dramatic and potentially detrimental conditions regarding the continued strain accumulation. As can be seen, the elevated frequencies quickly surpass the strain accumulation at the $1 \mathrm{~Hz}$ frequency by a little as 6000 cycles or less than 2 hours (at $1 \mathrm{~Hz}$ ).

This study indicates that increased strain accumulation can occur at elevated loading frequencies over prolonged time periods and do so indefinitely at elevated frequencies. Patients exposed to occupational vibrations in the $4 \mathrm{~Hz}$ to $20 \mathrm{~Hz}$ range experience low back pain rates twice that of non-exposed patients. Clinically, the mechanical response of the facet joints is dependent on a variety of specific loading parameters that include loading magnitude, duration and frequency and as a result low back pain is a multifaceted condition 
and cannot be attributed to a single factor. However, to minimize overall exposure, one must reduce amplitude, dampen frequency and reduce time. These parameters will all play a role in the overall exposure reduction. To that end, these effects can be reduced through improvements in seat materials, and damping or suspension mechanisms [16].

\section{Conclusions}

This study demonstrates that increased stiffness at elevated loading frequencies is manifested and may be an indicator of low back pain propensity. This study presents a mechanical representation of the facet joint response to high frequency vibration conditions and may be a basis for determination of low back pain predisposition. Further, subsequent reduction or damping of these elevated frequencies may aid in clinical care of those under continuous exposure.

\section{References}

[1] J.K. Freburger, G.M. Holmes, R.P. Agans, A.M. Jackman, J.D. Darter, A.S. Wallace, L.D. Castel, W.D. Kalsbeek and T.S. Carey, "The rising prevalence of chronic low back pain," Arch Intern Med, vol. 169, pp. 251-258, 2009.

[2] L. Manchikanti and V. Singh, "Review of Chronic Low Back Pain of Facet Joint Origin," Pain Physician, vol. 5, pp. 83-101, 2002.

[3] T. Waters, A. Genaidy, H. Barriera Viruet and M. Makola, "The impact of operating heavy equipment vehicles on lower back disorders," Ergonomics, vol. 51, pp. 602-636, 2008.

[4] D.G. Wilder, B.B Woodworth, J.W. Frymoyer and M.H. Pope, "Vibration and the Human Spine," Spine, vol. 7, pp. 243-254, 1982.

[5] S.E. Pinski, K.B. King, B.S. Davidson, B.H. Zhou, Y. $\mathrm{Lu}$ and $\mathrm{M}$. Solomonow, "High-frequency loading of lumbar ligaments increases proinflammatory cytokines expression in a feline model of repetitive musculoskeletal disorder," Spine J, vol. 10, pp. 10781085, 2010.

[6] M.H. Pope, M. Magnusson and D.G. Wilder, "Low back pain and whole body vibration," Clin Orthop Relat Res, vol. 354, pp. 241-248, 1998.

[7] B.L. Chen, Y.Q. Li, D.H. Xie and X.X. Yang, "LowMagnitude High-Frequency Loading via Whole Body Vibration Enhances Bone-Implant Osseointegration in Ovariectomized Rats," J Orthop Res, vol. 30, pp. 733739, 2012.
[8] A. Kaigle, L. Ekstrom, S. Holm, M. Rostedt and T. Hansson, "In vivo dynamic stiffness of the porcine lumbar spine exposed to cyclic loading: Influence of load and degeneration," J Spinal Disord, vol. 11, pp. 6570, 1998.

[9] L.X. Guo, E. Teo, K. Lee and Q. Zhang, "Vibration Characteristics of the Human Spine Under Axial Cyclic Loads: Effect of Frequency and Damping," Spine, vol. 30, pp. 631-7, 2005.

[10] A. Kettler, M. Bushelow and H.J. Wilke, "Influence of the loading frequency on the wear rate of a polyethylene-on-metal lumbar intervertebral disc replacement," Eur Spine J, vol. 21, pp. S709-S716, 2012.

[11] O. Izambert, D. Mitton, M. Thourot and F. Lavaste, "Dynamic stiffness and damping of human intervertebral disc using axial oscillatory displacement under a free mass system," Eur Spine J, vol. 12, pp. 5626, 2003.

[12] Y. Matsumotoa and M.J. Griffin, "Modeling the dynamic mechanisms associated with the principal resonance of the seated human body," Clin Biomech, vol. 16, pp. S31-S44, 2001.

[13] P. Zioupos, M. Gresle and K. Winwood. "Fatigue strength of human cortical bone: age, physical, and material heterogeneity effects," J Biomed Mater Res A, vol. 86, no. 3, pp. 627-636, 2008.

[14] J.I. Kåsin, N. Mansfield and A. Wagstaff, "Whole Body Vibration in Helicopters: Risk Assessment in Relation to Low Back Pain," Aviation, Space, and Environmental Medicine, vol. 82, pp. 790-796, 2011.

[15] T.E. Hill, G.T. Desmoulin and C.J. Hunter, "Is vibration truly an injurious stimulus in the human spine?," J Biomech, vol. 42, pp. 2631-2635, 2009.

[16] D.G. Wilder and M.H. Pope, "Epidemiological and etiological aspects of low back pain in vibration environments - an update," Clin Biomech, vol. 11, pp. 6173, 1996. 